\title{
Three Main Inducers of Alphacoronavirus Infection of Enterocytes: Sialic Acid, Proteases, and Low pH
}

\author{
Peng Yuan Zhou Yang Han Song Kai Wang Yang Yang Luyi Xie \\ Shilei Huang Jia Liu Lin Ran Zhenhui Song \\ Department of Veterinary Medicine, College of Animal Science, Southwest University, Chongqing, PR China
}

\section{Keywords}

Sialic acid · Protease · Low pH · Transmissible gastroenteritis virus · Porcine epidemic diarrhea virus · Porcine small intestine $\cdot$ Epithelial cells

\begin{abstract}
Transmissible gastroenteritis virus (TGEV) and porcine epidemic diarrhea virus (PEDV) are similar coronaviruses, causing diseases characterized by vomiting, diarrhea, and death from severe dehydration in piglets. Thus, they have caused huge losses to the swine-breeding industry worldwide. Nowadays, they are easily transmitted among the continents via vehicles, equipment, and cargo. Both viruses establish an infection in porcine enterocytes in the small intestine, and their spike (S) proteins play a key role in the virus-cell binding process under unfavorable conditions when the intestine with a low $\mathrm{pH}$ is filled with a thick layer of mucus and proteases. Sialic acid, proteases, and low $\mathrm{pH}$ are three main inducers of coronavirus infection. However, the details of how sialic acid and low pH affect virus binding to the host cell are not determined, and the functions of the proteases are unknown. This review emphasizes the role of three fac-
\end{abstract}

\section{KARGER}

(c) 2018 S. Karger AG, Basel

E-Mail karger@karger.com

www.karger.com/int tors in the invasion of TGEV and PEDV into porcine enterocytes and offers more insights into Alphacoronavirus infection in the intestinal environment.

(c) 2018 S. Karger AG, Basel

\section{Introduction}

Two porcine coronaviruses, transmissible gastroenteritis virus (TGEV) and porcine epidemic diarrhea virus (PEDV), are clustered as different species into the Alphacoronavirus genus. They both are important viral pathogens in piglets, causing similar pathological characteristics with acute diarrhea and dehydration $[1,2]$, leading to massive losses in the modern swine-breeding industry worldwide. Although their transmission route is limited to the fecal-oral route, as economic globalization increases rapidly and transportation develops remarkably, vehicles, equipment, and cargo have been convenient for these viruses to spread to all continents.

TGEV and PEDV replicate in enterocytes of the small intestine and are the causative agent of a fatal diarrhea in newborn piglets. Doyle and Hutchings [3] described the 
Table 1. Representative prototypes of coronaviruses with their own receptor or coreceptor

\begin{tabular}{lll}
\hline Genus & Representative prototypes & Receptor, coreceptor \\
\hline Alphacoronavirus & TGEV & pAPN, Neu5Ac, Neu5Gc \\
& HCoV-229E & hAPN \\
\hline Betacoronavirus & HCoV-NL63 & ACE2 \\
\hline & PEDV & pAPN, Neu5Ac \\
& SARS-CoV & DPP4 \\
Gammacoronavirus & BCoV & ACE2 \\
MHV & CEACAM1 \\
\hline Deltacoronavirus & HCoV-OC43 & Neu5, 9Ac2 \\
& IBV & Neu5Ac \\
& Bulbul coronavirus HKU11 \\
& Thrush coronavirus HKU12 & Unidentified \\
& Munia coronavirus HKU13 & \\
\hline
\end{tabular}

In the table, each color/line corresponding to one species is listed in the order as presented in the phylogenetic tree. The full names of the species in the table have been mentioned except for bovine coronavirus (BCoV) and human coronavirus OC43 (HCoV-OC43). The full names of the protein receptors and sugar receptors are shown in the table.

disease caused by TGEV in America for the first time. TGEV was then spreading to various continents: North America (Canada, 1989), Europe (England, 1957), and Asia (Japan, 1956, Korea, 2000, and Thailand, 2000). In China, TGEV was reported in the 1960s, and widespread outbreaks have occurred since 2010 [4]. In the 1970s, PEDV was first reported in Europe [5, 6]. Subsequently, the virus affected Asian countries extensively, and PEDV infection was usually mild before 2010 . The current virulent PEDV strains appeared in 2010 in China. Then, an outbreak occurred in Ohio in the USA in 2013, which spread throughout the USA. Since then, PEDV has resulted in significant economic damage worldwide and is thus receiving increased attention around the world [7].

TGEV and PEDV cluster in the genus Alphacoronavirus, which belongs to the Coronavirinae subfamily (Fig. 1) (order Nidovirales, family Coronaviridae). Coronaviruses (CoVs) are enveloped viruses with a singlestranded positive-sense RNA genome of up to approximately $30 \mathrm{~kb}$ with a $5^{\prime}$ cap and a $3^{\prime}$ polyadenylated tail [8]. They are capable of cross-species transmission and may gain new features. CoVs are significant infectious agents involved in gastroenteric, respiratory, hepatic, and neuronal diseases in animals and humans, causing cough and diarrhea, as well as high mortality rates, and bringing huge damage to human health and society [9]. $\alpha$ - and $\beta$-CoVs infect mammals such as humans and swine. By contrast, $\gamma$ - and $\delta$-CoVs are mostly detected in birds, such as Bulbul coronavirus HKU11, Thrush coronavirus
HKU12, and Munia coronavirus HKU13. Both $\gamma$ - and $\delta$-CoVs have also been found in mammals [10]. $\alpha-, \beta-$, $\gamma^{-}$, and $\delta$-CoVs are 4 genera of Coronaviridae that are clustered together based on numerous studies and serological and genotypic criteria (Table 1). Each genus has its own representative CoV species. TGEV, PEDV, and human coronaviruses (HCoV-229E and HCoV-NL63) are typical viruses of the genus $\alpha-\mathrm{CoV}$. The representative species of $\beta-\mathrm{CoV}$ are mouse hepatitis virus and severe acute respiratory syndrome coronavirus (SARS$\mathrm{CoV})$. Infectious bronchitis virus is currently the most studied virus in the $\gamma$-CoV genus [11]. Little is known about $\delta$-CoV.

This review emphasizes the role of three factors (sialic acid, proteases, and low $\mathrm{pH}$ ) in the invasion of TGEV and PEDV into porcine small intestine epithelial cells and provides information with respect to $a-\mathrm{CoV}$ infection that brings new insights into virus research.

\section{The Role of the Spike Protein of TGEV and PEDV}

The spike (S) protein of CoVs is essential for the interaction with receptors and the fusion of the viral particles and for cellular membranes. It also plays a crucial role in the interspecies transmission of CoVs. The interactions of CoV S glycoproteins with receptors on the cell surface determine the host range and tissue tropism of CoVs [12, 13]. Virus infection begins with the interplay between the 


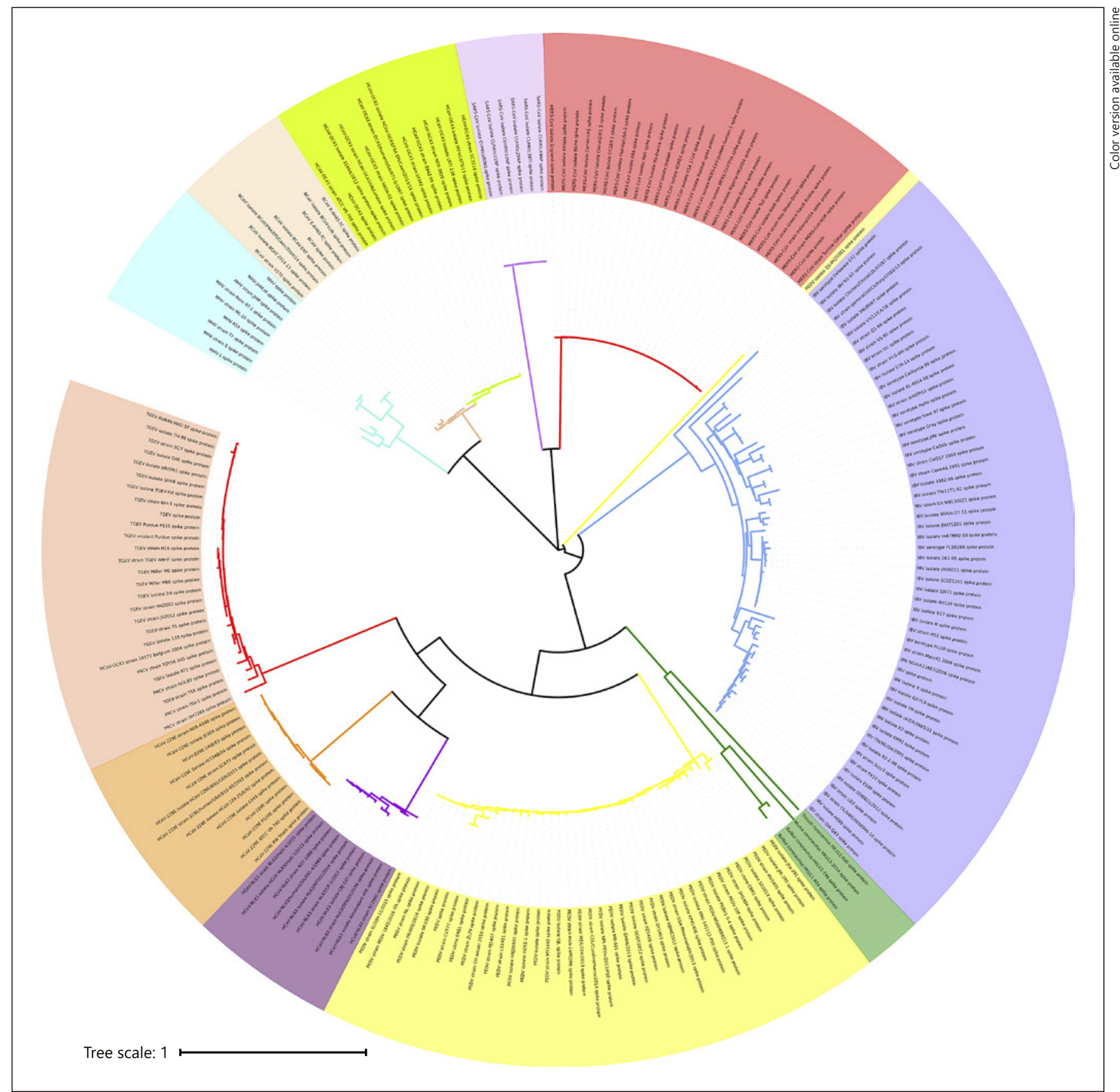

Fig. 1. Phylogenetic tree of the Coronavirinae subfamily. The phylogenetic tree was built on the basis of the nucleotide sequences of complete spike genes from 213 coronaviruses. The nucleotide sequence alignment and the construction of the phylogenetic tree were completed using the MEGA5.0 program with a proper substitution model: $\mathrm{d}=$ transitions + transversions, and all other settings were maintained as default. The final depiction of the phylo- genetic tree was completed using iTOL on the Internet. As the map shows, the coronavirus family is divided into 4 main groups, and 11 coronaviruses are regarded as representatives, which are shown in various colors. Interestingly, the spike gene of PEDV isolate strain ZJ14HZ030301 is separated into a single group in the phylogenetic tree. 
Fig. 2. Simplified graphic of the structural domains of the main coronaviruses' spike proteins. The spike protein structure can be divided into the S1 and S2 domains, and the structural domains in the spike protein are located in the order (from $\mathrm{C}$ to the $\mathrm{N}$ terminus) as: transmembrane (TM), heptad repeats (HRs) in the $\mathrm{S} 2$ domain, C-terminal domain (CTD), and N-terminal domain (NTD) in the S1 domain as well as the signal peptide (SP). In the graphic, compared with TGEV, PRCoV lacks an NTD, which is associated with enteric tropism. The RBD in the SARS spike protein is shown as discontinuous in the graphic.

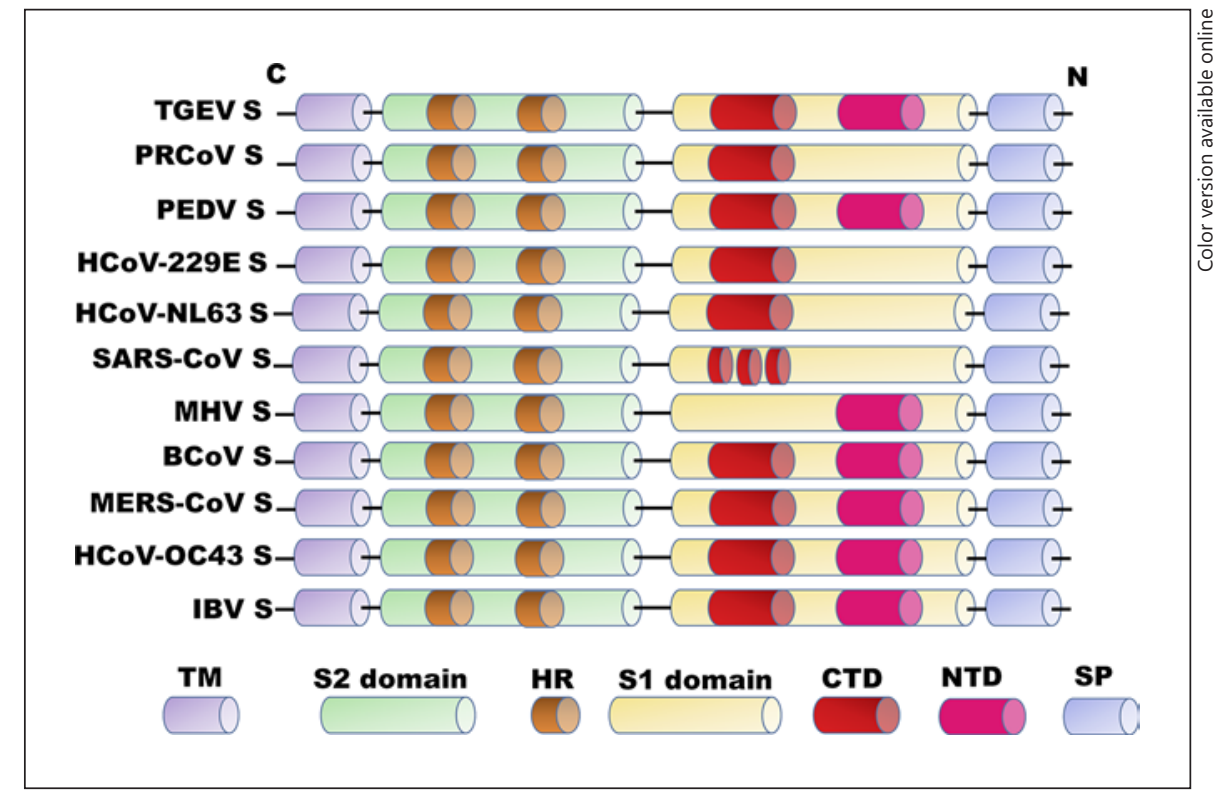

$S$ protein and its specific receptors, followed by penetration into the cells by a fusion event $[14,15]$.

The $\mathrm{S}$ protein, a class I fusion protein, is a membrane protein. It is the largest glycoprotein of CoVs [16], projecting out from the surface of $\mathrm{CoV}$ particles and forming a homotrimer structure called a peplomer. The $S$ protein is responsible for the corona-like appearance of the surface projections in the electron microscope. The peplomer includes a globular portion and a protein stalk. By adopting the helical structure that is characteristic of class I virus fusion proteins, the protein stalk connects the globular portion to the transmembrane domain [17]. The Nterminal S1 domain constitutes the globular region, and the stalk is made up of the membrane-proximal S2 domain. The N-terminal S1 domain and C-terminal S2 domain of the $\mathrm{S}$ protein play a similar role in all CoVs, the $\mathrm{S} 1$ region is related to receptor binding, and the S2 domain plays a role in the membrane fusion process. In addition, the S1 domain contains two subdomains, an $\mathrm{N}$ terminal domain (NTD) and a C-terminal domain (CTD) (Fig. 2). The two subdomains, called RBDs (receptor binding domains), bind with specific cell receptors, including a series of proteins and sugars. Thus, determinants in the S1 domain are not only crucial for initiating virus entry into cells, they also determine the cell and host tropism of CoVs [12].

TGEV and PEDV $S$ proteins have many similarities in their secondary structure. The TGEV S protein is produced as a 1,447-amino acid precursor polypeptide with a 16-residue signal peptide. According to analysis of the $S$ protein sequences of $\mathrm{CoVs}$ with different biological phenotypes, there are 4 antigenic sites (C, B, D, and A in that order) in the $\mathrm{N}$-terminal half of the $\mathrm{S}$ protein, among which the A site can induce neutralizing antibodies [4] and is highly conserved in TGEV and porcine respiratory coronavirus (PRCoV), a respiratory variant of TGEV. The S protein encoded by PRCoV lacks about 200 amino acids in the $\mathrm{N}$-terminal region that contain determinants related to the enteropathogenicity of TGEV [18]. PEDV has a 150 - to $220-\mathrm{kDa}$ spike glycoprotein with a homotrimeric structure. The PEDV $S$ protein is also a type I transmembrane glycoprotein that contains 5 regions, a signal peptide (residues 1-20), an S1 region (residues 21-793), an S2 region (residues 7941385), a transmembrane domain (residues 1335-1358), and a cytoplasmic tail (residues 1359-1385) [19]. The S1 region is responsible for virus particle binding to the cellular receptors, whereas the S2 region participates in membrane fusion of the virus and host cells. Like other CoVs, the $\mathrm{S} 1$ region possesses two subdomains comprising an NTD (residues 21-324) and a CTD (residues 253-638). The CTD of the S1 domain binds to a functional cellular receptor for PEDV infection. Sequence analyses of PEDV prototype and variant strains reveal that the $\mathrm{N}$ terminus of the $\mathrm{S}$ protein changes more easily than the $\mathrm{C}$ terminus. In addition, a previous report suggested an interaction of NTD of the S1 domain with a coreceptor $[20,21]$.
Yuan/Yang/Song/Wang/Yang/Xie/ Huang/Liu/Ran/Song 


\section{The Conformation of the RBD in TGEV and PEDV}

The a-CoV RBD comprises about 150 residues that adjoin the CTD in the $S 1$ region (Fig. 2). Previous studies on the CTD concluded that the structure had the characteristic of independent expression of the $S$ protein and preservation of its native structure maintained the binding specificity [4]. Several structural studies indicate that the $\alpha-\mathrm{CoV}$ RBD adopts a $\beta$-barrel fold with 2 highly twisted $\beta$-sheets, in which $3 \beta$-strands $(\beta 1, \beta 3$, and $\beta 7)$ run parallel and 3 disulfide bonds exist in the $3 \beta$-structures [22]. The RBD of TGEV is located within the CTD of the S1 domain [23]. In the TGEV RBD crystal structure, the bent-strand $\beta 5$ crosses both $\beta$-sheets. $\mathrm{N}$-linked glycans are concentrated at one side of the $\beta$-barrel; the opposite side is not glycosylated and might be closer to other $\mathrm{S}$ protein domains. The $\mathrm{N}$ - and $\mathrm{C}$-terminal ends of the RBD are located on the same side of the domain (terminal side); at the opposite side, $2 \beta$-turns form the tip of the barrel in the TGEV RBD [12].

Like TGEV, the crystal structure of a single domain unit in the PRCoV RBD adopts a $\beta$-barrel fold with 2 highly twisted $\beta$-sheets located in the CTD of the S1 domain and engages in binding to the host cell surface receptor. Compared with the TGEV S domain, the related PRCoV lacks the NTD, which is related to enteric tropism. The TGEV or PRCoV RBD tips consist of 2 protruding $\beta$-turns ( $\beta 1-\beta 2$ and $\beta 3-\beta 4$ ), each having a solvent-exposed aromatic residue (tyrosine or tryptophan) [20]. In the tertiary structure of the PRCoV RBD, 3 loops ( $\beta 1-\beta 2, \beta 3-\beta 4$, and $\beta 5-\beta 6)$ at the tips of the $\beta$-barrel domains are responsible for receptor binding. Some researchers found that single amino acid mutations in the 3 loops completely or significantly reduced the ability of PRCoV RBD to bind to host receptors, and mutations outside the RBD had no effect on receptor recognition [24].

To date, there have been few reports on the RBD structure of PEDV. It is interesting that 3 receptor binding mutant proteins, RBM1-1, RBM2-1, and RBM3-2 proteins, did not significantly reduce PEDV pAPN-binding activities in virus infection [4], suggesting the PEDV S protein uses a receptor-binding mechanism different from TGEV and PRCoV. PEDV is further confirmed to have a broader receptor range than other $\alpha-\mathrm{CoVs}$ [21].

\section{Cell Entry Receptors of TGEV and PEDV}

During the progress of evolution and adaptation to diverse hosts, CoVs have evolved to use various receptors to enter host cells. Different hosts or virus strains produce

Inducers of Alphacoronavirus Infection of Enterocytes evolutionary diversity in the same virus family, and the binding of CoVs to susceptible cells seems to show variation in the receptors used that correspond to viral groups and species. These $\mathrm{CoV}$ s recognize distinct cellular receptors and coreceptors, such as proteins and sugars, to facilitate their penetration into cells [8]. Currently, there are 4 main protein receptors: APN, angiotensin-converting enzyme 2 (ACE2), carcinoembryonic antigen-related cell adhesion molecule 1 (CEACAM1), and dipeptidyl peptidase 4 (DPP4). Most members of $\alpha-\mathrm{CoV}$ use APN as the receptor for infecting host cells, such as TGEV, PEDV, and $\mathrm{HCoV}-229 \mathrm{E}$. APN, also known as CD13, is a $150-\mathrm{kDa}$ type II transmembrane protein that belongs to a membrane-bound metalloprotease family [24]. Interestingly, $\mathrm{HCoV}-\mathrm{NL} 63$, as an $\alpha-\mathrm{CoV}$, shares the same cell entry receptor, identified as ACE2, with SARS-CoV, which is a $\beta$-CoV. ACE2 is a type I integral membrane glycoprotein with an $\mathrm{N}$-terminal extracellular domain comprising 2 a-helical lobes, between both of which there is a catalytic site with a coordinated zinc ion [25]. By contrast, the $\beta$-CoV mouse hepatitis virus utilizes CEACAM1 as a cell surface receptor for the S protein. CEACAM1, the first identified CoV receptor [26], is a type I transmembrane multifunctional protein and a member of the immunoglobulin superfamily termed IgSF. Middle East respiratory syndrome-related $\mathrm{CoV}$, belonging to $\beta-\mathrm{CoV}$, has been shown to use DPP4 as a cell entry receptor. DPP4 (also known as CD26), a type II membrane protein, is a multifunctional membrane-bound serine protease that forms homodimers on the surface of host cells. The DPP4 ectodomain comprises about 730 amino acids and has 2 domains, an $\alpha / \beta$-hydrolase domain and an 8-bladed propeller [27]. Among these cellular receptors and coreceptors, APN is a major cell entry receptor for CoVs. APN exists on the epithelial cell surface of different tissues. In particular, it is expressed abundantly in the brush border membrane of the small intestine, the kidney, and the respiratory tract [28]. Most $\alpha-\mathrm{CoV}$ s use APN as cell entry receptor. For example, previous studies showed that TGEV uses porcine (p) APN as the receptor in the entry process [24], whereas human (h) APN is a receptor for the infection by $\mathrm{HCoV}-229 \mathrm{E}$ [29].

The reason why TGEV uses APN as an entry receptor has not been clarified. It might be linked to its abundance on the surface of epithelial cells rather than its biological function, which seems to be dispensable for $\mathrm{CoV}$ binding capacity $[30,31]$. In the small intestine mucosa, APN occupies about $8 \%$ of the total protein content of the differentiated enterocytes [32]. For PEDV, although there are many articles in which pAPN was proposed as the recep- 
tor in PEDV infection, this view has been questioned due to the lack of robust direct evidence. The characteristic structure of APN is a large glycosylated ectodomain with a zinc metal ion at the active site, which functions as a zinc-dependent protease responsible for cleavage of the $\mathrm{N}$-terminal amino acids, mediated by the HELAH motif [33]. The enzymatic function of the pAPN catalyzes the removal of amino acid residues from the $\mathrm{N}$ termini of oligopeptides, and APN has been termed the "moonlighting enzyme" because of its many cell functions. APN can be cleaved into $\mathrm{N}$-terminal $(95 \mathrm{kDa})$ and C-terminal $(50$ $\mathrm{kDa}$ ) subunits by trypsin digestion and comprises 4 domains (DI-DIV) $[29,32]$. It is heavily glycosylated and forms dimers through extensive DIV-DIV interactions. Sequence conservation in the RBD tip of $\alpha-\mathrm{CoV}$ s exerts a crucial function in which the APN recognition mode is highly conserved [29]. Moreover, the specificity of APN with the recognition structure in the $S$ protein is linked to the structure of the APN N-linked glycan and fusion with the RBD $\beta 1-\beta 2$ turn. In addition, the CoVs tyrosine and tryptophan residues are critical in forming the TGEV RBD-APN structure. HCoV-229E does not have a tyrosine in its RBD $\beta 1-\beta 2$ turn, hence it recognizes the human APN that lacks this form of glycosylation [20,34], meaning that $\mathrm{HCoV}-229 \mathrm{E}$ recognition of APN must be unique. There have been some studies that determined the structure of a protruding tip for binding to small APN cavities in this human $\alpha-\mathrm{CoV}$. The $S$ proteins of PEDV and TGEV share high homology, but they have different host preferences. In addition, PEDV has been verified to use a different receptor recognition model compared with TGEV, $\mathrm{PRCoV}$, and HCoV-NL63. The N-terminal region in the PEDV S1 domain binds to sugars, which are regarded as its coreceptor [20].

\section{Sialic Acid Promotes TGEV and PEDV Binding to Host Cell Receptors}

In addition to binding to defined protein receptors, some CoVs show a sialic acid-binding activity. At present $\mathrm{CoVs}$ in $\alpha$-, $\beta$-, and $\gamma$-CoV have developed variant sialic acid binding activities [35]. According to current research, 3 types of sugars have been characterized as receptors or coreceptors for CoV entry into host cells: $5-\mathrm{N}$ acetylneuraminic acid (Neu5Ac), 5-N-glycolylneuraminic acid (Neu5Gc), and 5-N-acetyl-9-O-acetyl neuraminic acid (Neu5, 9Ac2) [36]. Recognition of sugars as co-receptors of TGEV and PEDV seems to be a strategy by which these viruses adapt to the living organism, meaning that TGEV and PEDV bind to sialic acid to survive under unfavorable intestinal tract conditions.

TGEV was first described to have a sialic acid binding activity in 1996 [37]. The sialic acid binding activity resides in the N-terminal portion of the S1 subunit that has been linked to the enteropathogenicity of TGEV and that is absent from the $\mathrm{S}$ protein of $\mathrm{PRCoV}$. The sialic acid preferentially recognized by TGEV is N-glycolylneuraminic acid (Neu5Ac) [37]. The spike protein has a trimeric structure and retains its sialic acid binding activity in soluble forms of the protein $[38,39]$. TGEV recognizes and binds the sugar moieties of glycoconjugates that are highly $\mathrm{O}$-glycosylated which promotes binding but is not sufficient for initiation of infection. It is believed that abundant sialic acid in mucins aids TGEV to penetrate the mucus layer and then to get access to pAPN on the surface of the intestinal epithelial cells. Thus, the efficiency of infection can be enhanced under unfavorable conditions. Binding to pAPN and sialic acid are two independent processes. Interestingly, binding to pAPN is more efficient in the absence of sialic acid [40].

There are few studies on the binding of PEDV to sialic acids. PEDV has been proven to have the ability to bind sialic acids. Neu5Ac presented the highest binding affinity with PEDV S1 in experiments using a glycan array screening. Moreover, the sialic acid binding region of PEDV was confirmed as being in the NTD of the $S$ protein (residues 1-320), similar to other CoVs [21]. However, it is unknown how the binding of sialic acid to the $S$ protein can affect PEDV entry into cells.

\section{The Function of Proteases during TGEV and PEDV Infection of Host Cells}

Increasing numbers of proteases have been demonstrated to participate in PEDV and TGEV infection of host cells in mechanisms where they do not act as receptors. These proteases are reportedly involved not only in adaptation of virus to innate immune response, but also in proteolytic processing of the $S$ protein.

$\mathrm{CoVs}$ always produce two types of cysteine proteases, a chymotrypsin-like main protease and papain-like proteases (PL1 pro and PL2pro). In general, they are essential for replicase polyprotein processing and viral replicationtranscription. PL1pro, a nonstructural protein of TGEV, resides in the nonstructural protein nsp3 subunits of TGEV [41]. The structure of TGEV PL1pro resembles a right hand with 3 distinct regions, the palm, thumb, and fingers (Fig. 3). It contains a zinc-binding domain, with 4
58

Intervirology 2018;61:53-63 DOI: $10.1159 / 000492424$
Yuan/Yang/Song/Wang/Yang/Xie/ Huang/Liu/Ran/Song 


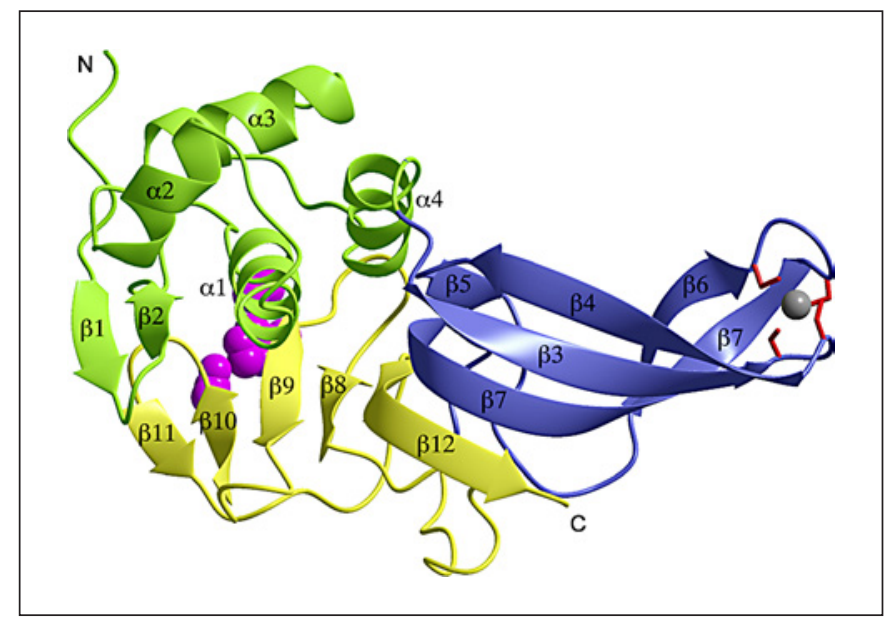

Fig. 3. Ribbon drawing of the TGEV PL1pro [40]. The entire structure resembles a hand, with the palm, thumb, and fingers represented in yellow, green, and blue, respectively. The catalytic triad is presented as magenta spheres, and the zinc ion is a gray sphere.

cysteine residues binding the zinc ion, and a catalytic triad formed by residues Cys32, His183, and Asp196 [4244]. The TGEV PL1pro protein, which contains a socalled USP-like binding site, exhibits deubiquitinating enzyme activity in vitro [45]. Deubiquitinating enzymes play essential roles in the innate immune response with interferon (IFN) secretion into the intestine mucosa.

In the intestine, the TGEV entry is restricted by IFNs in epithelial mucosa. The pathogen-associated molecular patterns of TGEV, virus RNAs, are sensed by pattern recognition receptors in host cells [46], triggering the IFN expression. TGEV arouses a early IFN- $\alpha$ production in intestinal secretions [47], IFN- $\alpha$ can improve B-cell response to provide protection against reinfection [48]. IFN- $\lambda$ is the main mucosal antiviral cytokine responsible for resisting virus invasion in the gut, such as TGEV and PEDV [49], together with interleukin-22, which forms the tissue barrier in intestinal epithelial cells to reduce viral infection [50].

TGEV PL1 protease possesses deubiquitinating activity and hydrolyzes the peptide that binds both Lys48- and Lys63-linked polyubiquitin chains [41]. Regulation of signaling molecules by ubiquitin has a significant function in the activation of the IFN response. TGEV PL1 binds and deubiquitinates retinoic acid-induced gene RIG1 and stimulator of interferon gene STING, which are regulators in the IFN signaling pathway, and then the levels of phosphorylated IFN regulatory factor 3 exhibit reduced activity [51], which counteracts IFN regulatory factor 3 translocating into the nucleus to activate the transcript of IFNs, such as IFN- $\alpha / \beta$ and IFN- $\lambda$. TGEV adopts the strategy to interfere and inhibit IFN secretion into the intestine gut to improve the efficiency of virus invasion.

Previous reports confirm that proteolytic processing of the $S$ protein contributes to the fusion of the viral membrane with cellular membranes and is necessary for virus entry. Proteases in virus entry are capable of cleaving the $S$ protein. These proteases in the pig small intestine potentially facilitate PEDV infection of host cells [52].

PEDV successfully infects African green monkey kidney (Vero) cells in vitro with extracellular trypsin [53]. The use of trypsin improves the possibility of PEDV entry, because trypsin facilitates $S$ protein-mediated fusion with the plasma membrane to deliver viral genomes into host cells. It has been shown that trypsin is helpful for syncytium formation in PEDV infection of MDCK cells [54] Interestingly, PEDV can also propagate without trypsin, suggesting that trypsin might be relevant for cellcell fusion rather than viral envelope-cell membrane fusion [55]. For PEDV entry into Vero cells under the trypsin-free conditions, endogenous proteases in endosome may adopt trypsin-like function, prompting PEDV S-mediated fusion.

TTSP is a type of trypsin-like serine protease termed type II transmembrane serine protease. TTSP is confirmed to cleave and activate proteins on the surface of influenza viruses and CoVs, allowing multicycle replication in the absence of trypsin. TTSPs are reportedly involved in the release of PEDV virions [56]. TMPRSS2 and MSPL are members of TTSPs. TMPRSS2 and MSPL exhibit trypsin-like features in the amplification of PEDV in vitro in the absence of trypsin and play a vital role in cellcell fusion and virus-cell fusion. It is found that PEDV $S$ protein is colocalized with TMPRSS2 and MSPL extensively and cleaved by coexpression with TMPRSS2 or MSPL. TMPRSS2 and MSPL could cleave PEDV S protein into two fragments of the same size. The two TTSPs interact with the PEDV S protein to promote viral entry into cells by promoting cell-cell fusion and virus-cell fusion. Moreover, MSPL exhibited the strongest effect in the replication of PEDV compared to TMPRSS2. Interestingly, the adaptive capacity and the growth of PEDV in Vero cells expressing TMPRSS2 and MSPL are higher than those in cells treated with trypsin [57].

It is comfirmed that PEDV requires serine and serinelike proteases (Fig. 4) for its entry through endocytosis in the early stages of infection. For cell-cell fusion, serine proteases are involved in PEDV entry in an acidic $\mathrm{pH}-$ independent manner [58]. Cellular serine proteases pos- 


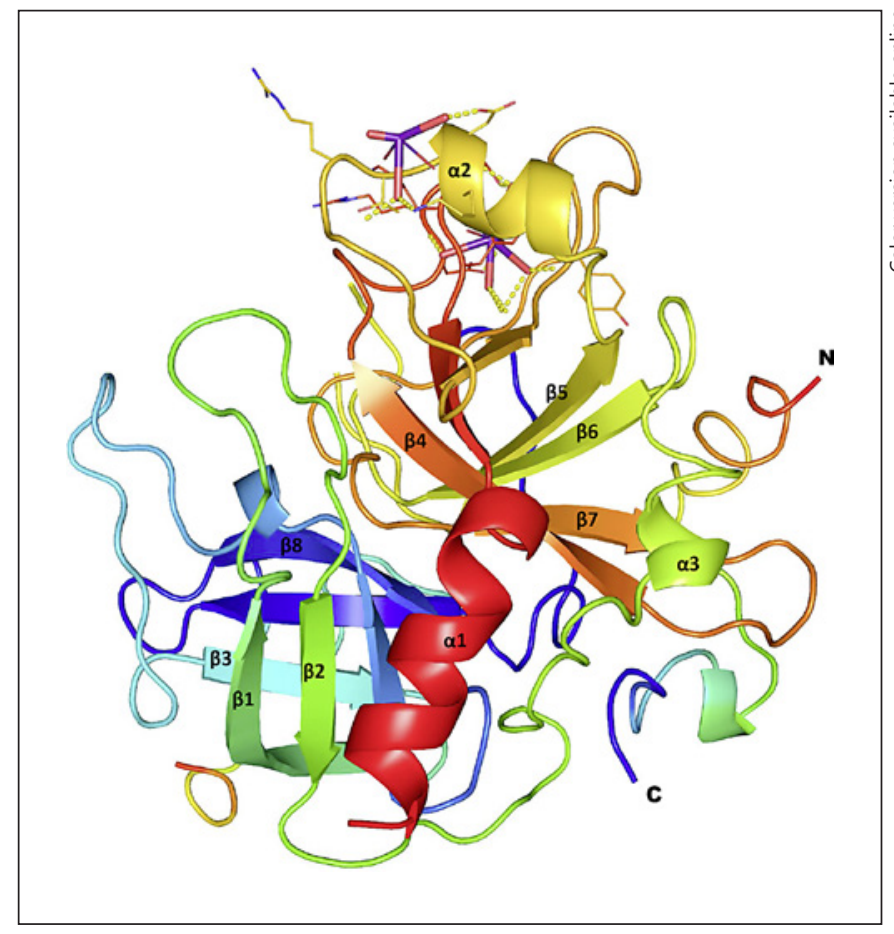

Fig. 4. Ribbon drawing of the serine protease (PBD ID 1hxe). The $\mathrm{X}$-ray diffraction method was used to test the structure. The structure comprises $4 \alpha$-helixes $(\alpha 1-\alpha 4)$ and $8 \beta$-sheets $(\beta 1-\beta 8)$ presented in diverse colors in the light of different domains. There is an $\mathrm{Na}^{+}$site close to $\beta 2$ (yellow), shown in stick form.

sess a catalytic triad of amino acids comprising His, Asp, and Ser, which are located in similar positions at the 3-dimensional structure. The nucleophilic Ser is responsible for cleavage and is often replaced by a functionally and spatially equivalent Cys in viral trypsin-like proteases [42, 44]. The Asp of the active-site residues can be substituted by an equivalent Glu. Serine or serine-like proteases in the cytoplasm are required for the fusion between the PEDV envelope and the host endosomal membrane. Serine proteases activate PEDV entry in a low $\mathrm{pH}$-independent manner.

New evidence has emerged that pAPN in the small intestine acts as a protease for PEDV, which contrasts with the idea that pAPN plays role as a cell surface receptor. The structure of APN is shown in Figure 5. With regard to the similarities between PEDV and TGEV and the accordance of the pathology of PEDV infection with the tissue distribution of pAPN, pAPN used to be regarded as a receptor for PEDV, which was supported by some indirect evidence [24, 59]. However, PEDV can also infect and propagate in pAPN-negative Vero cells $[53,58]$, indicating that there is no direct evidence to support the view that $\mathrm{pAPN}$ is a receptor for PEDV.

In current research, by employing CPK cells to express porcine homologs of pAPN, ACE2, CEACAM1, and DPP4, results were obtained showing that CPK cells were susceptible to TGEV, but not to PEDV. In addition, PEDV infection was not affected by soluble pAPNs, suggesting that PEDV utilizes different receptors compared with other CoVs. By contrast, another study showed that when pAPN was overexpressed in porcine CPK cells, pAPN enabled PEDV multiplication [59, 60]. Another study showed that nonpermissive ST cells expressing a pAPN gene supported productive infection of PEDV of the host cells, showing that constitutive overexpression of pAPN directly promotes PEDV multiplication [29]. In summary, pAPN more likely functions as a protease in PEDV infection rather than as a receptor, which is supported by research evidence; however, the mechanism by which PAPN promotes PEDV infection remains unknown.

\section{Structural Changes and Activation of TGEV and PEDV Proteins at Low pH}

It is unquestionable that $\mathrm{pH}$ is important for the process of CoV entry into cells. Low $\mathrm{pH}$ is necessary for conformational changes of viral glycoproteins and proteolytic activation of viral glycoproteins by endosomal proteases [61]. It is reported that $\mathrm{pH}$-dependent conformational changes occur in the $\mathrm{CoV}$ peplomer [62], and the S1 domain is released from peplomers on the surface of the virus. Inside the endosome, endogenous proteases participating in membrane fusion are active in a low-pH environment. TGEV and PEDV tend to multiply at a low $\mathrm{pH}$. The amount of TGEV in the medium at pH 6.5 was 10 -fold greater than that at $\mathrm{pH} 7.2$, and the yield was almost 100-fold higher than that at $\mathrm{pH} 8.0$ [63].

TGEV binds to APN and enters the host cell via endocytosis. Upon attaching to the porcine APN receptor, TGEV is incorporated into the cell membrane and enters by way of caveola-dependent endocytosis [64]. Subsequent membrane fusion is promoted by active cellular proteases in the endosome in a low-pH environment [65, 66]. Using MDCK cells overexpressing porcine APN, it has been demonstrated that acidification of the intracellular compartment promotes membrane fusion inside the endosome and cellular proteases are activated in a $\mathrm{pH}$ dependent manner to facilitate membrane fusion $[59,65]$.
60

Intervirology 2018;61:53-63 DOI: $10.1159 / 000492424$
Yuan/Yang/Song/Wang/Yang/Xie/ Huang/Liu/Ran/Song 


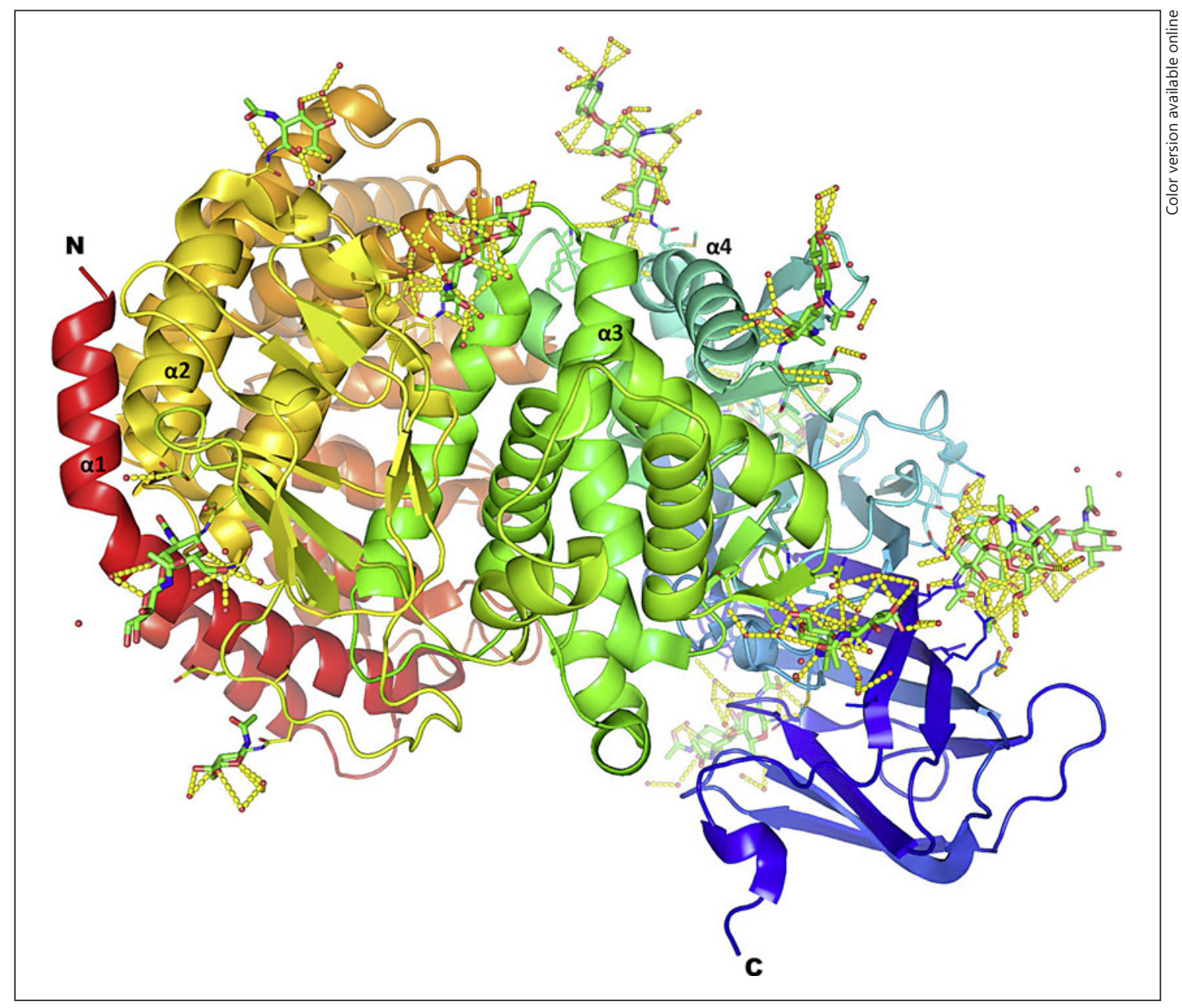

Fig. 5. Ribbon drawing of the pAPN structure (PBD ID 4FKE). The structure was well characterized using X-ray diffraction. The pAPN structure contains 4 main domains in the order: N-terminal DI (red), DII (yellow), DIII (green), and C-terminal DIV (blue).

Different from the caveola-mediated uptake of TGEV, PEDV enters cells using endocytosis via clathrin-coated pits. After the first step of interacting with receptors on the cell surface, PEDV penetration is facilitated by viral envelope fusion with the host cell plasma membrane, which happens inside a $\mathrm{pH}$-dependent endosomal compartment. The endosomal cellular elements are very important for the activation of low $\mathrm{pH}$ dependent proteases, rather than the virus needing an acidic environment to trigger its entry [41, 67]. The acidic conditions do not affect PEDV itself, suggesting that acidic $\mathrm{pH}$ is not the only factor that affects viral infection of host cells.

Inducers of Alphacoronavirus Infection of Enterocytes

\section{Conclusions}

Interaction between the S protein and the host cells is an indispensable step for viral entry into host cells. In the process of TGEV and PEDV entry into cells, the environmental conditions in the intestinal gut are complex and unfavorable. A thicker layer of mucus containing sialic acid covers the intestinal epithelium, and the small intestine generally has a low $\mathrm{pH}$. In addition, the intestine is filled with proteases from the stomach and intestinal wall. These conditions make it hard for viruses to infect intestinal cells compared with infection of cultured cells. The selection of TGEV and PEDV RBDs interacting with cell surface receptors is the result of pressure from immune 
surveillance. Sialic acid usage by TGEV and PEDV promotes the efficient invasion into host cells. PEDV and TGEV invade enterocytes in the intestinal epithelium by exploiting proteases in the small intestine. TGEV induces PL1pro to hijack cellular canonical pathways to prevent viral protein degradation, facilitating later virus entry into targeted cells. TMPRSS2 and MSPL interact with the PEDV S protein to promote viral entry into cells by promoting cell-cell fusion and virus-cell fusion. Recently, porcine APN has been observed to function as a protease for PEDV, not as a receptor, to promote viral multiplication during PEDV entry into the cell, the mechanism of which requires further study. Low $\mathrm{pH}$ in the initial stage of PEDV entry into intestinal epithelial cells allows structural changes and protease activation during binding to surface receptors.

\section{Acknowledgments}

We thank Zhangcheng Li (College of Animal Science, Southwest University) for his guidance in constructing the evolutionary tree. This project has been supported by the Fundamental Research Funds for the Central Universities (XDJK2017D082).

\section{Disclosure Statement}

No potential conflict of interest is reported by the authors.

\section{References}

1 Kim L, Hayes J, Lewis P, et al: Molecular characterization and pathogenesis of transmissible gastroenteritis coronavirus (TGEV) and porcine respiratory coronavirus (PRCV) field isolates co-circulating in a swine herd. Arch Virol 2000;145:1133-1147.

2 Song D, Park B: Porcine epidemic diarrhoea virus: a comprehensive review of molecular epidemiology, diagnosis, and vaccines. Virus Genes 2012;44:167-175.

3 Doyle LP, Hutchings LM: A transmissible gastroenteritis in pigs. J Am Vet Med Assoc 1946;108:257-259.

4 Reguera J, Ordoño D, Santiago C, et al: Antigenic modules in the $\mathrm{N}$-terminal $\mathrm{S} 1$ region of the transmissible gastroenteritis virus spike protein. J Gen Virol 2011;92:1117-1126.

5 Chasey D, Cartwright SF: Virus-like particles associated with porcine epidemic diarrhoea. Res Vet Sci 1978;25:255-256.

6 Pensaert MB, de Bouck P: A new coronaviruslike particle associated with diarrhea in swine. Arch Virol 1978;58:243-247.

7 Wang D, Fang L, Xiao S: Porcine epidemic diarrhea in China. Virus Res 2016;226:7-13.

8 Masters PS: The molecular biology of coronaviruses. Adv Virus Res 2006;66:193-292.

9 Schwegmann-Wessels C, Bauer S, Winter C, et al: The sialic acid binding activity of the $S$ protein facilitates infection by porcine transmissible gastroenteritis coronavirus. Virol J 2011;8:435.

10 Woo PC, Lau SK, Lam CS: Discovery of seven novel mammalian and avian coronaviruses in the genus deltacoronavirus supports bat coronaviruses as the gene source of alphacoronavirus and betacoronavirus and avian coronaviruses as the gene source of gammacoronavirus and deltacoronavirus. J Virol 2012;86: 3995-4008.
11 Reguera J, Mudgal G, Santiago C, et al: A structural view of coronavirus-receptor interactions. Virus Res 2014;194:3-15.

12 Belouzard S, Millet JK, Licitra BN, et al: Mechanisms of coronavirus cell entry mediated by the viral spike protein. Viruses 2012;4:10111033.

13 Li F: Structure, Function, and Evolution of Coronavirus Spike Proteins. Annu Rev Virol 2016;3:237-261.

14 Li F: Receptor recognition mechanisms of coronaviruses: a decade of structural studies. J Virol 2015;89:1954-1964.

15 Gallagher TM, Buchmeier MJ: Coronavirus spike proteins in viral entry and pathogenesis. Virology 2001;279:371-374.

16 Wang J, Deng F, Ye G, et al: Comparison of lentiviruses pseudotyped with $S$ proteins from coronaviruses and cell tropisms of porcine coronaviruses. Virol Sin 2016;31:49-56.

17 Beniac DR, Andonov A, Grudeski E, et al: Architecture of the SARS coronavirus prefusion spike. Nat Struct Mol Biol 2006;13:751752.

18 Sánchez CM, Izeta A, Sánchez-Morgado JM, et al: Targeted recombination demonstrates that the spike gene of transmissible gastroenteritis coronavirus is a determinant of its enteric tropism and virulence. J Virol 1999; 73:7607-7618.

19 Lee DK, Cha SY, Lee C: The N-terminal region of the porcine epidemic diarrhea virus spike protein is important for the receptor binding. Korean J Microbiol Biotechnol 2011; 39:140-145.

20 Liu C, Tang J, Ma Y, et al: Receptor usage and cell entry of porcine epidemic diarrhea coronavirus. J Virol 2015;89:6121-6125.

21 Deng F, Ye G, Liu Q, et al: Identification and comparison of receptor binding characteris- tics of the spike protein of two porcine epidemic diarrhea virus strains. Viruses 2016;8: 55.

22 Reguera J, Santiago C, Mudgal G, et al: Structural bases of coronavirus attachment to host aminopeptidase $\mathrm{N}$ and its inhibition by neutralizing antibodies. PLoS Pathog 2012; 8:e1002859.

23 Wu K, Li W, Peng G, et al: Crystal structure of NL63 respiratory coronavirus receptorbinding domain complexed with its human receptor. Proc Natl Acad Sci USA 2009;106: 19970-19974.

24 Delmas B, Gelfi J, L'Haridon R, et al: Aminopeptidase $\mathrm{N}$ is a major receptor for the enteropathogenic coronavirus TGEV. Nature 1992; 357:417-420

25 Li W, Moore MJ, Vasilieva N, et al: Angiotensin-converting enzyme 2 is a functional receptor for the SARS coronavirus. Nature 2003;426:450-454

26 Williams RK, Jiang GS, Holmes KV: Receptor for mouse hepatitis virus is a member of the carcinoembryonic antigen family of glycoproteins. Proc Natl Acad Sci USA 1991;88: 5533-5536.

27 Raj VS, Mou H, Smits SL, et al: Dipeptidyl peptidase 4 is a functional receptor for the emerging human coronavirus-EMC. Nature 2013;495:251-254.

28 Godet M, Grosclaude J, Delmas B, Laude H: Major receptor-binding and neutralization determinants are located within the same domain of the transmissible gastroenteritis virus (coronavirus) spike protein. J Virol 1994;68: 8008-8016

29 Yeager CL, Ashmun RA, Williams RK, et al: Human aminopeptidase $\mathrm{N}$ is a receptor for human coronavirus 229E. Nature 1992;357: $420-422$. 
30 Li W, Zhang C, Sui J, et al: Receptor and viral determinants of SARS-coronavirus adaptation to human ACE2. EMBO J 2005;24:16341643.

31 Wentworth DE, Tresnan DB, Turner BC, et al: Cells of human aminopeptidase N (CD13) transgenic mice are infected by human coronavirus-229E in vitro, but not in vivo. Virology 2005;335:185-197.

32 Kenny AJ, Maroux S: Topology of microvillar membrance hydrolases of kidney and intestine. Physiol Rev 1982;62:91-128.

33 Rangel R, Sun Y, Guzman-Rojas L, et al: Impaired angiogenesis in aminopeptidase $\mathrm{N}$ null mice. Proc Natl Acad Sci USA 2007;104: 4588-4593.

34 Tusell SM, Schittone SA, Holmes KV: Mutational analysis of aminopeptidase $\mathrm{N}$, a receptor for several group 1 coronaviruses, identifies key determinants of viral host range. J Virol 2007;81:1261-1273.

35 Hoffmann M, Müller MA, Drexler JF, et al: Differential sensitivity of bat cells to infection by enveloped RNA viruses: coronaviruses, paramyxoviruses, filoviruses, and influenza viruses. PLoS One 2013;8:e72942.

36 Schwegmann-Wessels C, Herrler G: Sialic acids as receptor determinants for coronaviruses. Glycoconj J 2006:23:51-58.

37 Schultze B, Krempl C, Ballesteros ML, et al: Transmissible gastroenteritis coronavirus, but not the related porcine respiratory coronavirus, has a sialic acid (N-glycolylneuraminic acid) binding activity. J Virol 1996;70: 5634-5637.

38 Shahwan K, Hesse M, Mork AK, et al: Sialic acid binding properties of soluble coronavirus spike (S1) proteins: differences between infectious bronchitis virus and transmissible gastroenteritis virus. Viruses 2013;5:19241933.

39 Schwegmann-Wessels C, Herrler G: Identification of sugar residues involved in the binding of TGEV to porcine brush border membranes. Methods Mol Biol 2008;454:319-329.

40 Malykh YN, King TP, Logan E, et al: Regulation of N-glycolylneuraminic acid biosynthesis in developing pig small intestine. Biochem J 2003;370:601-607.

41 Wojdyla JA, Manolaridis I, van Kasteren PB, et al: Papain-like protease 1 from transmissible gastroenteritis virus: crystal structure and enzymatic activity toward viral and cellular substrates. J Virol 2010;84:10063-10073.

42 Dessens JT, Lomonossoff GP: Mutational analysis of the putative catalytic triad of the cowpea mosaic virus $24 \mathrm{~K}$ protease. Virology 1991;184:738-746.
43 Dougherty WG, Parks TD, Cary SM, et al: Characterization of the catalytic residues of the tobacco etch virus $49-\mathrm{kDa}$ proteinase. Virology 1989;172:302-310.

44 Margis R, Pinck L: Effects of site-directed mutagenesis on the presumed catalytic triad and substrate-binding pocket of grapevine fanleaf nepovirus 24-kDa proteinase. Virology 1992; 190:884-888

45 Bhoj VG, Chen ZJ: Ubiquitylation in innate and adaptive immunity. Nature 2009;458: 430-437.

46 Kawai T, Akira S: Toll-like receptors and their crosstalk with other innate receptors in infection and immunity. Immunity $2011 ; 34: 637-$ 650.

47 La Bonnardiere C, Laude H: High interferon titer in newborn pig intestine during experimentally induced viral enteritis. Infect Immun 1981;32:28-31.

48 Deal EM, Lahl K, Narvaez CF, et al: Plasmacytoid dendritic cells promote rotavirus-induced human and murine B cell responses. J Clin Invest 2013;123:2464-2474.

49 Zhang Q, Yoo D: Immune evasion of porcine enteric coronaviruses and viral modulation of antiviral innate signaling. Virus Res 2016;226: 128-141.

50 Xue M, Zhao J, Ying L, et al: IL-22 suppresses the infection of porcine enteric coronaviruses and rotavirus by activating STAT3 signal pathway. Antiviral Res 2017;142:68-75.

$51 \mathrm{Hu}$ X, Tian J, Kang H, et al: Transmissible gastroenteritis virus papain-like protease 1 antagonizes production of interferon- $\beta$ through its deubiquitinase activity. Biomed Res Int 2017;2017:7089091.

52 Zamolodchikova TS: Serine proteases of small intestine mucosa - localization, functional properties, and physiological role. Biochemistry 2012;77:820-829.

53 Hofmann M, Wyler R: Propagation of the virus of porcine epidemic diarrhea in cell culture. J Clin Microbiol 1988;26:2235-2239.

54 Li BX, Ge JW, Li YJ: Porcine aminopeptidase $\mathrm{N}$ is a functional receptor for the PEDV coronavirus. Virology 2007;365:166-172.

55 Wicht O, Li W, Willems L, et al: Proteolytic activation of the porcine epidemic diarrhea coronavirus spike fusion protein by trypsin in cell culture. J Virol 2014;88:7952-7961.

56 Shirato K, Matsuyama S, Ujike M: Role of proteases in the release of porcine epidemic diarrhea virus from infected cells. J Virol 2011;85:7872-7880.
57 Shi W, Fan W, Bai J, et al: TMPRSS2 and MSPL facilitate trypsin-independent porcine epidemic diarrhea virus replication in Vero cells. Viruses 2017;9:E114.

58 Kusanagi K, Kuwahara H, Katoh T, et al: Isolation and serial propagation of porcine epidemic diarrhea virus in cell cultures and partial characterization of the isolate. J Vet Med Sci 1992;54:313-318.

59 Sturman LS, Ricard CS, Holmes KV: Conformational change of the coronavirus peplomer glycoprotein at $\mathrm{pH} 8.0$ and $37 \mathrm{de}$ grees $\mathrm{C}$ correlates with virus aggregation and virus-induced cell fusion. J Virol 1990; 64:3042.

60 Cong Y, Li X, Bai Y, et al: Porcine aminopeptidase $\mathrm{N}$ mediated polarized infection by porcine epidemic diarrhea virus in target cells. Virology 2015;478:1-8.

61 Park JE, Cruz DJ, Shin HJ: Clathrin- and serine proteases-dependent uptake of porcine epidemic diarrhea virus into Vero cells. Virus Res 2014;191:21-29.

62 Weismiller DG, Sturman LS, Buchmeier MJ, et al: Monoclonal antibodies to the peplomer glycoprotein of coronavirus mouse hepatitis virus identify two subunits and detect a conformational change in the subunit released under mild alkaline conditions. J Virol 1990; 64:3051-3055.

63 Pocock DH, Garwes DJ: The influence of $\mathrm{pH}$ on the growth and stability of transmissible gastroenteritis virus in vitro. Arch Virol 1975; 49:239-247.

64 Weingartl HM, Derbyshire JB: Evidence for a putative second receptor for porcine transmissible gastroenteritis virus on the villous enterocytes of newborn pigs. J Virol 1994;68: 7253-7259.

65 Hansen GH, Delmas B, Besnardeau L, et al: The coronavirus transmissible gastroenteritis virus causes infection after receptor-mediated endocytosis and acid-dependent fusion with an intracellular compartment. J Virol 1998; 72:527-534.

66 Paul A, Trincone A, Siewert S, et al: A lysinemethionine exchange in a coronavirus surface protein transforms a retention motif into an endocytosis signal. Biol Chem 2014;395:657665

67 Simmons G, Gosalia DN, Rennekamp AJ, et al: Inhibitors of cathepsin L prevent severe acute respiratory syndrome coronavirus entry. Proc Natl Acad Sci USA 2005;102:1187611881. 